\title{
Dark Matter Detector Delivers Enigmatic Signal
}

\author{
Are the excess events detected by the XENON1T experiment a harbinger of \\ new physics or a mundane background?
}

\author{
By Tongyan Lin
}

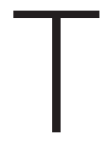
he dark matter that pervades the Universe appears to be fairly inert, which is why it's so hard to find clues to its nature. Now, an unexpected result from the XENON1T experiment is setting off a wave of excitement among dark matter hunters. Over the past fifteen years, the XENON Collaboration has designed one of the quietest and darkest laboratory environments on Earth-the perfect setting to spot an elusive dark matter particle. The collaboration has now reported unexpected flashes of light that can't easily be explained as background noise, but also don't line up with expectations based on popular dark matter models [1]. The prospect of a profound discovery has already set off a burst of activity aimed at explaining these tantalizing results (see Synopsis: Theorists React to Potential Signal in Dark Matter Detector).

XENON's main goal has always been to detect a particular type of dark matter particle, called a weakly interacting massive particle (WIMP). In a deep underground laboratory below the Gran Sasso massif in Italy, the collaboration prepared an enormous tank of purified liquid xenon surrounded by photomultiplier tubes. If a WIMP flying through the tank knocked a xenon atom hard enough, it could produce detectable light (Fig. 1). XENON and similar experiments have put enormous effort into controlling background noise that could look like a signal, reducing, in particular, the contribution from traces of radioactive isotopes in the setup or the environment.

The new results come from the most sensitive version of this experiment-XENON1T, with 2 tons of ultrapure xenon in the tank. In data taken over one year, the researchers found 285 detection events in an energy range where they expected only
232 background events-an excess with a 3.5 sigma statistical significance. The events, however, don't look like they came from WIMPs. An atom recoiling after being hit by a WIMP should release both photons and electrons. Since WIMPs interact with the atoms' nuclei, the expected electron-to-photon ratio is small [2]. The large ratio of the reported events indicates that they come from particles that interact with the atoms' electrons.

The XENON researchers analyzed three possible explanations for their data: particles emitted from the Sun, dark matter bosons that behave differently from WIMPs, and traces of radioactive contaminants.

The core of the Sun is a nuclear reactor burning at 15 million kelvin, producing a bath of particles with enough energy to create a signal like the one in XENON1T. Most of these particles would be trapped by the surrounding dense matter in the stellar core. But particles that interact only feebly with matter could stream out of the Sun. This explanation may have nothing to do with dark matter, but it might require particles or interactions beyond those we know.

Neutrinos-a byproduct of fusion in the Sun's core-are the first possibility. They could easily reach XENON1T's detector but aren't expected to produce much of a signal there. The signals, however, could be explained if neutrinos have a larger magnetic moment than predicted by the standard model. The second possibility is a new type of particle, the axion, which could be produced in the Sun through interactions with electrons, photons, or nuclei. This scenario is appealing because these axions would solve an unrelated, yet important puzzle known as the strong CP problem. Both the nonstandard neutrinos and the solar axion hypotheses, however, are in conflict with 


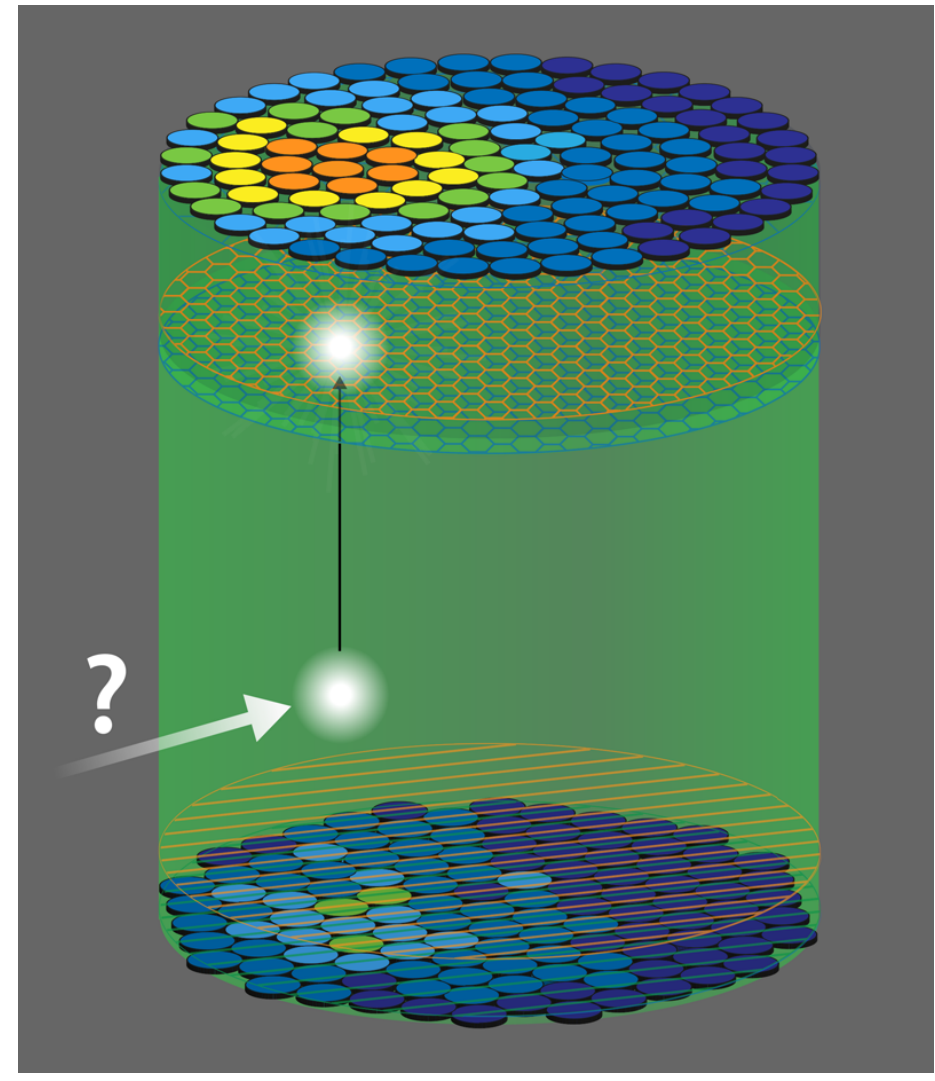

Figure 1: An incoming particle hitting atoms in XENON1T's tank releases photons and electrons that can be detected by photomultipliers at the top and bottom of the tank. The collaboration has reported excess signals that can't easily be explained in terms of standard model particles or background noise $[\mathbf{1}]$.

Credit: XENON1T; adapted by APS/Alan Stonebraker

observations of stellar populations: If these particles were emitted by the Sun in sufficient numbers to explain the XENON1T signal, they would be emitted by other stars, too, and this emission would accelerate their cooling. One of the consequences is that there would be many fewer bright white dwarfs than observed $[3,4]$.

The most intriguing explanation is that XENON1T is seeing signs of dark matter, just not the WIMPs it was looking for. While a WIMP would collide with or scatter from an atom, a bosonic dark matter particle could be absorbed by the atom [5]. Similar to the photoelectric effect, in which absorbed photons generate photoelectrons, the absorption of a boson could release an electron from the atom. In this case, the energy of the collision should correspond to the dark matter particle mass. Two promising dark matter candidates with masses around $2 \mathrm{keV}$ are consistent with this explanation. The first is an axion with a mass larger than that of the above-mentioned solar axions. The second is a dark photon, a heavier cousin of conventional photons with a much weaker interaction with matter. The XENON Collaboration estimates that if dark photons were absorbed at a rate $10^{-30}$ times smaller than regular photons, they could explain the signal.

A significant wrench in all exotic-physics interpretations is the possible contribution from backgrounds that are difficult to pin down. Through years of work, XENON has achieved unprecedented background suppression, but some faint, unwanted radioactivity remains. Part of this background can be ascribed to different isotopes of xenon, krypton, iodine, and lead, whose presence can be quantified by independent measurements. But other contributions, such as that of tritium, aren't well understood. If the detector contains just three tritium atoms per kilogram of xenon, the beta decay of tritium alone could explain the signal. The researchers acknowledge that they lack information to rule out this scenario. It might well be that these ton-scale dark matter experiments have become so sensitive that they pick up this tiny contamination that can't be independently tested.

With a 3.5 sigma significance, the signal is worth paying attention to but is not conclusive. But we shouldn't have to wait too long to see if any of these interpretations holds up.

XENONnT, the successor of XENON1T with a larger tank and a lower background, is in the works. Two similar experiments, LUX-ZEPLIN in the U.S. and PandaX in China, will weigh in as well. Since a radioactive background likely depends on the detector, finding a consistent signal across multiple experiments would be remarkable. What's more, collecting more detection events would allow researchers to build more precise energy spectra for the events, which could allow them to vet different hypotheses based on the expected spectra.

The ideas surrounding this result show how much the dark matter landscape is growing. A flurry of studies is already putting forth many other particle physics ideas as explanations. Some of those explore ways to reconcile the solar particle explanation with observations of stellar populations. Others 
point out that the signal could be explained by dark matter theories involving multiple particles with different masses. Come what may, the new result is an exciting step into the unknown.

Tongyan Lin: Department of Physics, University of California, San Diego, La Jolla, CA, USA

\section{REFERENCES}

1. E. Aprile et al. (XENON Collaboration), "Excess electronic recoil events in XENON1T,” Phys. Rev. D 102, 072004 (2020).

2. E. Aprile et al. (XENON Collaboration), "Dark matter search results from a one ton-year exposure of XENON1T," Phys. Rev. Lett. 121, 111302 (2018).

3. M. M. Miller et al., "Revisiting the axion bounds from the Galactic white dwarf luminosity function," J. Cosmol.

Astropart. Phys. 2014, 069 (2014).

4. S. Arceo Díaz et al., "Constraint on the axion-electron coupling constant and the neutrino magnetic dipole moment by using the tip-RGB luminosity of fifty globular clusters," arXiv:1910.10568.

5. M. Pospelov et al., "Bosonic super-WIMPs as keV-scale dark matter," Phys. Rev. D 78, 115012 (2008). 